\title{
Adaptation, linguistic and clinimetric validation of the Bangla version of Zarit Burden Interview
}

\author{
Farzana Rabin, Jhunu Shamsun Nahar, Mohammad S. I. Mullick, Helal Uddin Ahmed and Nafia \\ Farzana
}

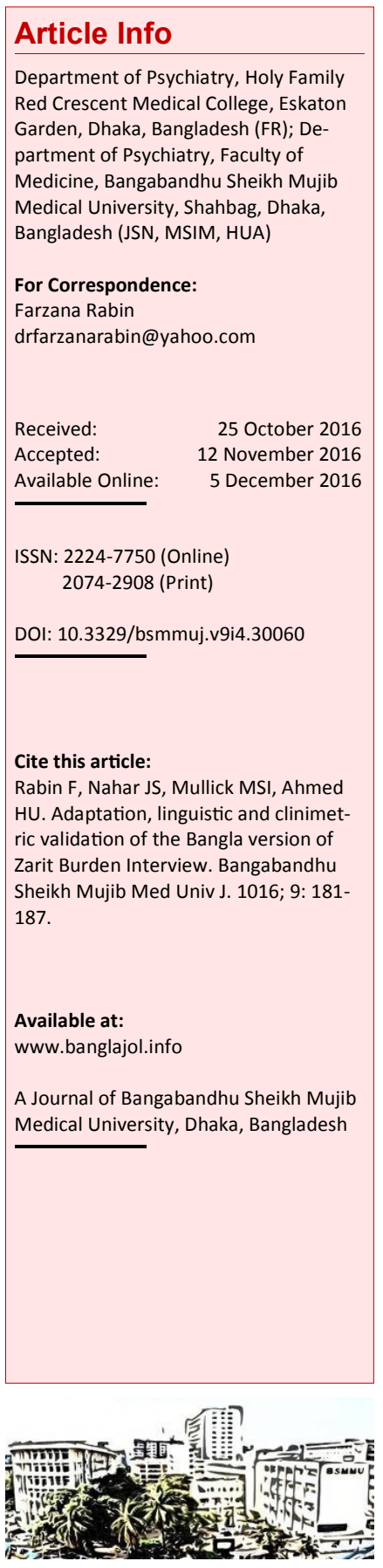

\section{Abstract}

The aim of this study was to develop a culturally adapted and validated Bangla version of Zarit Burden Interview (ZBI-B) questionnaire for use in Bangla speaking caregiver of patient with dementia. This study was conducted on 100 caregivers related to consecutively attending outpatients with a previously established primary diagnosis of dementia, according to DSM-IV criteria. Validity and reliability were evaluated by comparing with the caregiver burden inventory (CBI). An exploratory factor analysis with the principle component with varimax rotation was used to detect the factorial structure in observed measurements. To attain the best-fitting structure and the correct number of factors, the following criteria were used: Eigen values $>1.0$, factor loadings $>0.30$. The Cronbach's alpha value was 0.847 for test and 0.839 retest. The intra-class correlation for the test-retest reliability was 0.89 . The ZBI score was highly correlated with the CBI score (Pearson's correlation coefficient, $r=0.909, p=0.001$ ). From the exploratory factor analysis six factors comprising 20 items were extracted with Eigen values higher than 1.00 accounting for $69 \%$ of the total item variance. In conclusion, ZBI-B is valid, reliable and useful for use in clinical contexts and in future studies that could lead to a better understanding of caregiver burden in dementia.

\section{Introduction}

Psychiatric patients need assistance or supervision in their daily activities and this often places a major burden on their caregivers, thereby placing the caregiver at a great risk of mental and physical health problems.1 Considering the health impact of caregiving, it is very important to recognize and quantify the burdens upon family caregivers with some appropriate predictor or tools.,2,3 Identifying the challenges and potential impacts of caregiving will facilitate the plan of management or care of the patient.

Several scales are already developed to measure caregiver burden based on experience of different country. $\cdot, 9-12$ The Zarit Burden Interview (ZBI), which provides a comprehensive assessment of both objective and subjective burden, is one of the most commonly used burden measures and has been validated in many culturally or ethnically different populations. The ZBI was primarily developed to measure subjective burden among the caregivers of people with dementia. $\underline{4}$

There is still no validated instrument to measure the caregiver burden in Bangladesh.
Considering the huge future mass of patients with dementia in Bangla speaking people it demands a widely used, reliable, valid and responsive instrument in Bangla language to assess caregiver burden. Hence, the purpose of this study was to validate the Bangla version of the ZBI (ZBI-B) by examining its internal consistency, test-retest reliability, and concurrent validity in family caregivers of patients with dementia in Bangladesh.

\section{Materials and Methods}

\section{Questionnaire}

A semi-structured questionnaire was prepared to study of adaptation, linguistic and clinimetric validation of the Bangla version of $\mathrm{ZBI}$. It contained the socio-demographic variables like age, sex, habitat, religion, educational status, occupation, monthly family income, marital status, type of family, etc. It also contains some relevant caregiving related variables like relationship with the patients, whether he/she was the main caregiver, staying in the same residence, having previous experience of caregiving and the duration of caregiving. 


\section{ZBI-B}

The ZBI is a 22 item instrument for measuring the caregiver's perceived burden of providing family care. 4 The 22 items are assessed on a 5 point Likert scale, ranging from $0=$ 'never' to $4=$ 'nearly always'. Item scores are added up to give a total score ranging from 0 to 88 , with higher scores indicating greater burden. The questions focus on major areas such as caregiver's health, psychological well-being, finances, social life and the relationship between the caregiver and the patient.

\section{Bangla version of caregiver burden inventory}

Burden of caregivers was also assessed with another instrument "Caregiver burden inventory" (CBI). 12 The investigator administered the inventory by reading the statement and marking the responses. It is a 24 item multi-dimensional questionnaire measuring caregiver burden with 6 subscales: a) Time dependence; b) Developmental; c) Behavior; d) Physical burden; e) Social burden; and f) Emotional burden. Scores for each item were evaluated using a 5 point Likert scale ranging from 0 (not at all disruptive) to 4 (very disruptive).

\section{Procedure of the data collection}

One hundred diagnosed cases of dementia were selected from the Dementia Clinic at the Neurology and Psychiatry Outpatient Department. One of his/ her selected caregivers was enrolled. When more than one caregiver of a particular patient were attended, who contributed more was selected by the researcher. After taking informed written consent, the socio-demographic information of patient and caregiver was documented in the questionnaire to identify the socio-demographic characteristics by face to face interview.

The caregivers were asked to self-administer the ZBI-B questionnaire that comprised questions assessing demographics and some standardized instruments including the ZBI. Although the questionnaire was designed to be self-administered, the subject was first given an explanation and rundown on the questionnaire by the investigator before being left to complete the questionnaire. The same caregiver was also asked to fill-up the questionnaire second time 2 weeks after completion of first survey for the assessment of test-retest reliability. Another scale CBI was also used by investigator to test the validity by asking questions and filling up the response.

\section{Cross-cultural adaptation $\underline{13}$}

Stage I: The ZBI questionnaire was translated initially by two persons (T1 by a medical person and $\mathrm{T} 2$ by a person who had no medical or clinical background).

Stage II: Two translations were synthesized into one translation (T-12).

Stage III: This part (T-12) was translated back to the original (English) (BT1 and BT2) by two individuals who had competency on both Bangla and English.

Stage IV: To consolidate all the versions of the questionnaire and to develop what would be considered the prefinal version of the questionnaire for field testing an expert committee was formed. The committee was composed of methodologists, health professionals (psychiatrist and clinical psychologists), language professionals, and the translators (forward and backward translators) involved in the process up to this point. The committee reviewed all the translations and reach a consensus on any discrepancy. The material at the disposal of the committee includes the original questionnaire, and each translation (T1, T2, T12, BT1, BT2) together with corresponding written reports (which explain the rationale of each decision at earlier stages).

Stage V (Pretesting): The final stage of adaptation process was the pretest. This field test of the new questionnaire used the prefinal version in 30 subjects or patients from the Neurology and Psychiatry Outpatient Department. Each subject completed the questionnaire, and was interviewed to probe about what he or she thought was meant by each questionnaire item and the chosen response. Both the meaning of the items and responses were explored.

\section{Assessment of validity and reliability of ZBI-B}

Caregivers $(n=100)$ aged $\geq 18$ years attending patients with dementia were evaluated thoroughly. All were asked for occupation, level of education and social status. ZBI-B questionnaire was served individually. The reliability of the ZBI-B was assessed by test-retest reliability test, the concurrent validity and face validity were seen by comparing with CBI.

\section{Ethical issue}

Patients and all caregivers were informed about the purpose of the study and ethical issues were explained. The study protocol was approved by Institutional Review Board of Bangabandhu Sheikh Mujib Medical University. We have contacted the author and the permission was taken for validation of its ZBI-B.

\section{Results}

Mean ZBI score of individual item ranged from 0.1 \pm 0.4 to $2.1 \pm 0.9$. The highest score was $2.1 \pm 0.9$ for the item 7 "Are you afraid what the future holds for your relative?" followed by item 21 "Do you feel you could do a better job in caring for your relative?" (1.9 \pm 1.1$)$ and item 20 "Do you feel you should be doing more for your relative?" (1.9 \pm 1.0$)$ (Table I). Total ZBI-B score ranged from 10.0 to 61.0 


\section{Table I}

\section{Distribution of scores of individual ZBI items for individual item}

$\begin{array}{lll}\text { SL. } & \text { ZBI item } & \text { Mean } \pm \text { SD }\end{array}$

No.

Do you feel that your relative asks for more help than he/she needs?

2 Do you feel that because of the time you spend with your relative that you don't have enough time for yourself?

3 Do you feel stressed between caring for your relative and trying to meet other responsibilities for your family or work?

$4 \quad$ Do you feel embarrassed over your relative's behavior?

5 Do you feel angry when you are around your relative?

6 Do you feel that your relative currently affects your relationship with other family members or friends in a negative way?

$7 \quad$ Are you afraid what the future holds for your relative?

8 Do you feel your relative is dependent upon you?

9 Do you feel strained when you are around your relative?

10 Do you feel your health has suffered because of your involvement with your relative?

11 Do you feel that you don't have as much privacy as you would like, because of your relative?

12 Do you feel that your social life has suffered because you are caring for your relative?

13 Do you feel uncomfortable about having friends over, because of your relative?

14 Do you feel that your relative seems to expect you to take care of him/her, as if you were the only one he/she could depend on?

15 Do you feel that you don't have enough money to care for your relative, in addition to the rest of your expenses?

16 Do you feel that you will be unable to take care of your relative much longer?

17 Do you feel you have lost control of your life since your relative's illness?

18 Do you wish you could just leave the care of your relative to someone else?

19 Do you feel uncertain about what to do about your relative?

20 Do you feel you should be doing more for your relative?

21 Do you feel you could do a better job in caring for your relative?

22 Overall, how burdened do you feel in caring for your relative?
$1.4 \pm 0.9$

$1.5 \pm 1.2$

$1.0 \pm 1.1$

$1.8 \pm 1.0$

$0.7 \pm 0.9$

$1.1 \pm 0.9$

$2.1 \pm 0.9$

$1.5 \pm 1.3$

$0.7 \pm 1.0$

$0.6 \pm 0.9$

$0.8 \pm 1.0$

$0.9 \pm 0.9$

$1.2 \pm 1.0$

$1.7 \pm 1.5$

$1.2 \pm 1.1$

$1.0 \pm 1.0$

$0.1 \pm 0.4$

$0.7 \pm 0.9$

$1.5 \pm 1.0$

$1.9 \pm 1.0$

$1.9 \pm 1.1$

$1.6 \pm 1.0$
Table II

Distribution of total burden score according to $\mathrm{ZBI}$ and $\mathrm{CBI}$ in test and retest

\begin{tabular}{|c|c|c|}
\hline Variables & Mean \pm SD & Range \\
\hline ZBI for test & $27.3 \pm 11.2$ & $10.0-61.0$ \\
\hline CBI for test & $19.9 \pm 13.2$ & $4.0-58.0$ \\
\hline ZBI for retest & $27.2 \pm 10.8$ & $6.0-56.0$ \\
\hline CBI for retest & $20.0 \pm 12.2$ & $3.0-54.0$ \\
\hline
\end{tabular}

(mean $27.3 \pm 11.2$ ) for test and 6.0 to 56.0 (mean 27.2 \pm 10.8 ) and total burden in CBI ranged from 4.0 to 58.0 (mean $19.9 \pm 13.2$ ) for test and 3.0 to 54.0 (mean $19.9 \pm 12.2$ ) (Table II). ZBI-B score in test $35.0 \%$ of caregivers have little or no burden, $54.0 \%$ have mild to moderate burden, $10.0 \%$ have moderate to severe burden and $1.0 \%$ have severe burden. In retest $31.6 \%$ of caregivers have little or no burden, $58.9 \%$ have mild to moderate burden, $9.5 \%$ have moderate to severe burden and none have severe burden (Table III). The ZBI Intra Class Correlation (ICC) and Cronbach's alpha reflected a good reliability $(>0.7)$ (Table IV). In principle, component analysis six components Eigen values greater than one and this six components explain the total variance is 68.9 (Table V).

\section{Table III}

Distribution of caregivers according to degree of their burden in test retest reliability ZBI

$$
\text { Test Retest }
$$$$
(\mathrm{n}=100) \quad(\mathrm{n}=95)
$$

\begin{tabular}{|lr|r|}
\hline Little or no burden & 35 & 30 \\
Mild to moderate burden & 54 & 56 \\
Moderate to severe burden & 10 & 9 \\
Severe burden & 1 & 0 \\
\hline
\end{tabular}

\section{Table IV}

ZBI intraclass correlation and Cronbach's alpha

Variables

Intraclass correlation

95\% Confidence interval

$0.7-0.9$

Cronbach's alpha for test

0.8

Cronbach's alpha for retest

0.8

\section{Reliability and validity}

The Cronbach's alpha value was 0.847 for test and 


\begin{tabular}{|c|c|c|c|c|c|c|}
\hline \multicolumn{7}{|c|}{ Table V } \\
\hline \multicolumn{7}{|c|}{ Analysis of principle components } \\
\hline \multirow{2}{*}{$\begin{array}{l}\text { Compo- } \\
\text { nent }\end{array}$} & \multicolumn{3}{|c|}{ Initial Eigen values } & \multicolumn{3}{|c|}{ Rotation sums of squared loadings } \\
\hline & Total & \%Variance & \%Cumulative & Total & \%Variance & \%Cumulative \\
\hline 1 & 6.5 & 29.5 & 29.5 & 4.4 & 20.0 & 20.0 \\
\hline 2 & 3.0 & 13.8 & 43.3 & 2.7 & 12.5 & 32.5 \\
\hline 3 & 1.8 & 8.3 & 51.6 & 2.4 & 10.8 & 43.2 \\
\hline 4 & 1.4 & 6.4 & 58.0 & 2.0 & 9.2 & 52.6 \\
\hline 5 & 1.3 & 6.0 & 64.0 & 2.0 & 8.9 & 61.4 \\
\hline 6 & 1.1 & 4.9 & 68.9 & 1.6 & 7.4 & 69.0 \\
\hline
\end{tabular}
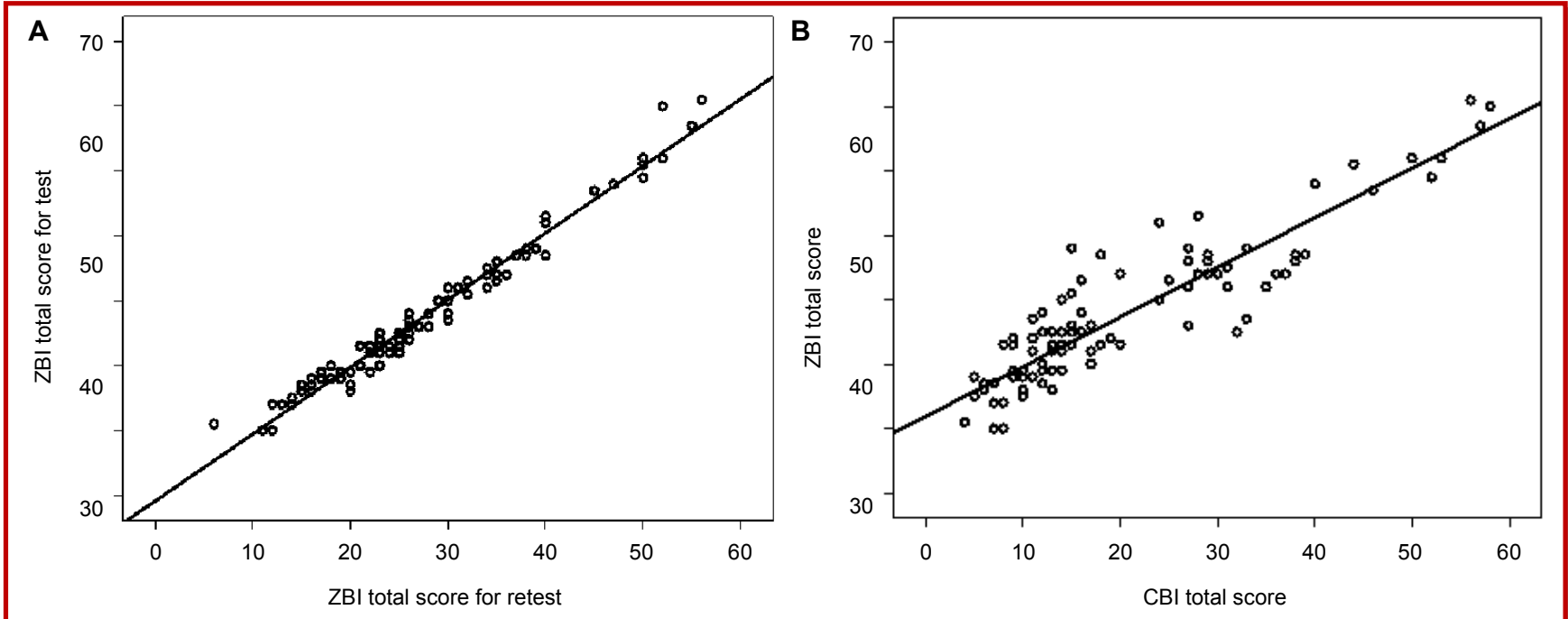

Figure 1: (A) Correlation of ZBI-B test score with ZBI-B retest score. Pearson's correlation show that ZBI-B score is significantly correlated with its retest score. (B) Correlation of ZBI-B score with CBI score. Pearson's correlation show that ZBI-B score is significantly correlated with CBI score

0.839 retest which was examined with 95 subjects. The intra-class correlation for the test-retest reliability, which was examined with 95 subjects, was 0.89 (When interpreting Cronbach's alpha or the intra class $R$, a value $\geq 0.7$ reflects good reliability).

\section{Construct validity}

The Zarit Burden score was highly correlated with the CBI score (pearson's correlation coefficient, $r=$ $0.909, \mathrm{p}=0.001$ ) (Figure 1).

\section{Factor analysis}

Exploratory factor analysis on the ZBI-B was performed based on the principle component method with a varimax rotation, to detect the factor structure in the observed variables. From the exploratory factor analysis six factors comprising 20 items were extracted with Eigen values higher than
1.00 accounting for $69 \%$ of the total item variance. Seven items $(1,2,8,10,14,15$ and 22) indicating "time and financial dependence or burden due to dependence" are loaded on the first factor accounting for $30 \%$ of the total variance in the matrix. The second factor (items 11, 12, 13) representing "deprivation of personal time and social burden" accounted for the $14 \%$ of the variance. The third (items 21 and 20) component indicating "burden due to guilt feelings" accounted for $9 \%$ of the variance. The fourth factor (items 7 and 19) "burden due to excessive concern/ working/uncertainity" accounted for $6 \%$ of the variance. The fifth (items 18, 16, 17) "burden due to excessive stress/stress burden" accounted for $6 \%$ of variance, and Sixth factor (items 4, 5, 6) "emotional burden" accounts for $5 \%$ of variance. Item 3 and 9 do not represent any factor in original ZBI version and not suitable in the Bangla version. 


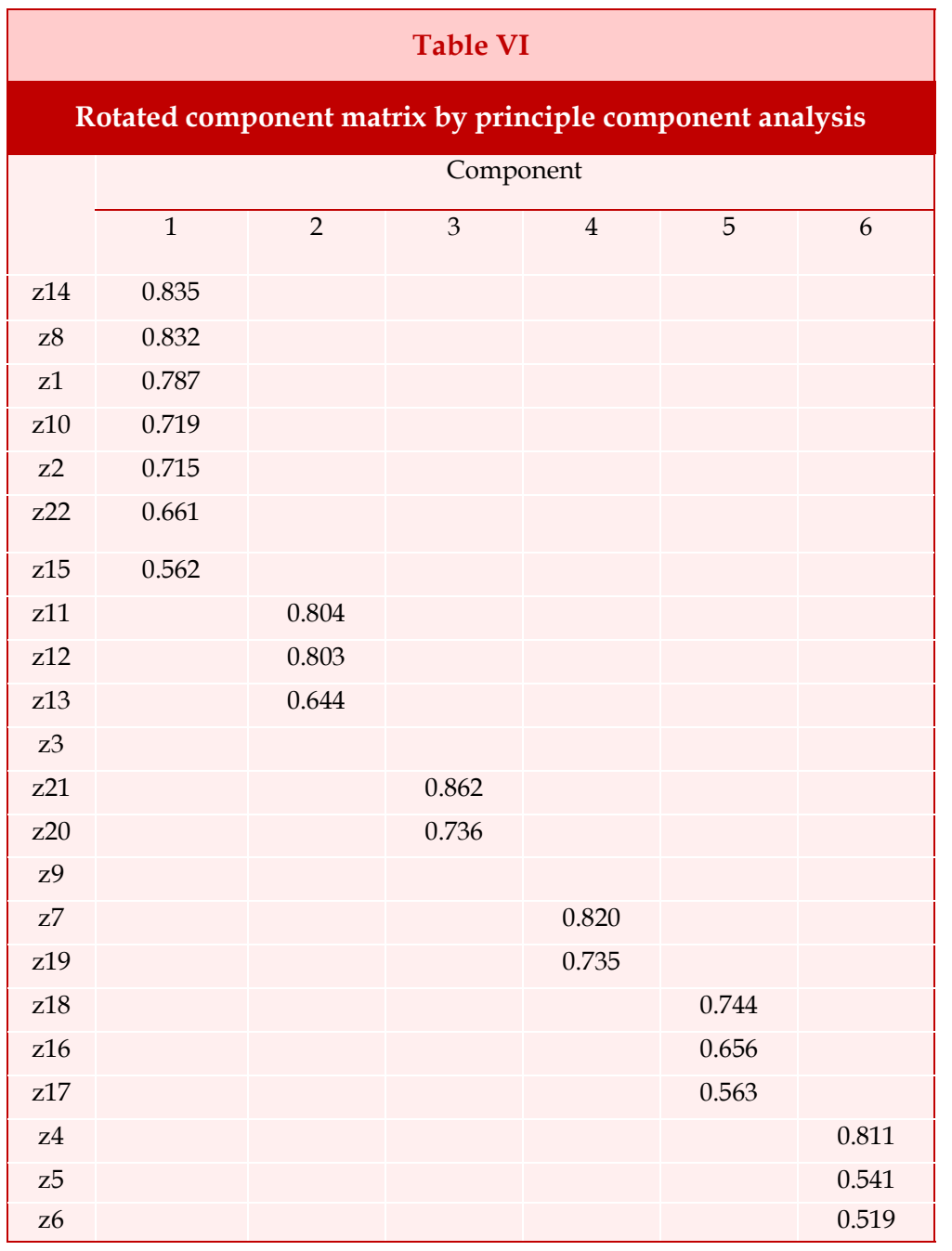

\section{Discussion}

The ZBI has been translated into many languagesChinese,,$\underline{14}$ Japanese, $\underline{15}$ Italian, $\underline{16}$ Korean, $\underline{17}$ German, $\underline{18}$ Brazilian $\underline{19}$ and Turkish $\underline{20}$ and has been adopted for assessing caregiver burden in many different patient populations, for example dementia, cancer populations, psychiatric illness, multiple sclerosis, stroke, Parkinson's disease and chronic obstructive pulmonary disease. Currently, a Bangla version of the ZBI is not available and the present study therefore aims to validate the Bangla version of the 22 item ZBI (ZBI-B), evaluating its reliability, calculating its internal consistency and concurrent validity. It was studied on 100 caregiver of people with dementia measuring caregiver burden with ZBI-B and comparing that with CBI (caregivers burden inventry). $\underline{12}$

In the current study minimum and maximum total ZBI-B score was 10.0 and 61.0 and mean score of $27.3 \pm 11.2$ for test and $27.2 \pm 10.8$ for retest, which are comparable to those reported by Zarit et al. $\underline{4}$ and other validation studies. $16,21-24$ "Are you afraid what the future holds for your relative?" item 7 had highest mean ZBI score, similarly in previous studies it had high burden score.16,18,21,24 In those studies item 8 "I feel the patient is too dependent" had highest burden. In the current study, item 21 "Do you feel you could do a better job in caring for your relative?" had the second highest score and followed by item 20 "Do you feel you should be doing more for your relative?". ZBI-B score in test $35.0 \%$ of caregivers have little or no burden, $54.0 \%$ have mild to moderate burden, $10.0 \%$ have moderate to severe burden and $1.0 \%$ have severe burden. In retest $31.6 \%$ of caregivers have little or no burden, $58.9 \%$ have mild to moderate burden, 9.5\% have moderate to severe burden and none have severe burden. A high Cronbach's a coefficient indicated that the internal consistency of ZBI was good. The Cronbach's alpha value was 0.847 for test and 0.839 retest which was examined with 95 subjects. These are comparable to other version of ZBI like Cronbach's a 0.875 for Chinese version, $\underline{\mathbf{1 4}}$ 0.88 for Japanese version, $\underline{\mathbf{1 5}} 0.90$ for Italian version, $\underline{\mathbf{1 6}}$ 0.80 for Turkish version 20 and 0.92 for German version 18 . The intra class correlation for the testretest reliability, which was examined with 95 subjects, was 0.89 (When interpreting Cronbach's alpha or the intra class $R$, a value $\geq 0.70$ reflects good reliability). Caregiver's burden is a multifaceted composition and a global score may not provide a complete and accurate assessment. Caregivers with an identical score may be affected by different aspects of burden. $\underline{25}$ As obtained by different authors, $\underline{23,26}$ the factorial structure seems to be composed of more than two factors. Part of this confusion might derive from the fact that different authors have used different factor analytical methods or have included different subsets of the 22 ZBI items. Exploratory factor analysis on the ZBI-B was performed based on the principle component method with a varimax rotation, to detect the factor structure in the observed variables. From the exploratory factor analysis six factors comprising 20 items were extracted with Eigen values higher than 1.00 accounting for $69 \%$ of the total item variance (V). Seven items $(1,2,8,10,14,15$ and 22) indicating "time and financial dependence or burden due to dependence" are loaded on the first factor accounting for $30 \%$ of the total variance in the matrix. The second factor (items 11, 12, 13) representing "deprivation of personal time and social burden" accounted for the $14 \%$ of the variance; the third (items 21 and 20) component indicating "burden due to guilt feelings' accounted for $9 \%$ of the variance; the fourth factor (items 7 and 19) "burden due to excessive concern /working/ uncertainity" accounted for $6 \%$ of the variance; the fifth (items 18, 16, 175) "burden due to excessive stress/tress burden" accounted for $6 \%$ of variance and sixth factor (items 4,5,6) "emotional burden" 
accounts for $5 \%$ of variance. Item 3 and 9 of the do not represent any factor in original ZBI version and not suitable in the Bangla version. In previous studies for Chinese version five factors had an Eigen value greater than 1.00 and accounted for $56.4 \%$ of total variance, for Italian version five factors comprising all 22 items were extracted with Eigen values higher than 1.00 accounting for $60 \%$ of the total item variance. İnci and Erdem 27 determined their Turkish version of the ZBI had a three-factor structure with an Eigen value of $>1$. In the current study, the first factor accounts for the majority of the variance and has the majority of the items loading on to it, followed by the second factor. Even if this instrument shows a high internal consistency it seems to have a multi-factorial structure. The reports of high internal consistency and multiple subfactors are not necessarily contradictory, because psychometric theory shows that a multi-factorial scale can achieve high a levels if the items are evenly apportioned across the subscales. Two items ( 3 and 9 ) have not been found to be fit for any factor of ZBI-B.

Pearson correlation for test-retest reliability of ZBI$B$ was $r=0.896(p=0.001)$. The acceptable minimum point for test-retest reliability is 0.70 according to previous literature. $\underline{28}$ The Zarit Burden score was highly correlated with the CBI score (Pearson's correlation coefficient, $\mathrm{r}=0.909, \mathrm{p}=0.001$ ).

\section{Conclusion}

The results of this study indicate good psychometric properties of the ZBI-B.

\section{References}

1. Ampalam P, Gunturu S, Padma V. A comparative study of caregiver burden in psychiatric illness and chronic medical illness. Indian J Psychiatry. 2012; 54: 239-43.

2. National Alliance for Caregiving. Caregiving in the U.S. 2009. Retrieved from http://www.caregiving.org/data/Caregiving_in_the _US_2009_full_report.pdf. [Accessed on 18/10/16].

3. Stucki BR, Mulvey J. Can aging baby boomers avoid the nursing home? Long-term care insurance for aging in place. Washington DC, American Council of Life Insurers, 2000, p 15.

4. Zarit SH, Reever KE, Bach-Peterson J. Relatives of the impaired elderly: Correlates of feelings of burden. Gerontologist 1980; 20: 649-55.

5. Carretero S, Garces J, Rodenas F, Sanjose V. The informal caregiver's burden of dependent people: Theory and empirical review. Arch Gerontol
Geriatr. 2009; 49: 74-79.

6. Gouin JP, Hantsoo L, Kiecolt-Glaser JK. Immune dysregulation and chronic stress among older adults a review. Neuroimmunomodulation 2008; 15: 251-59.

7. Lee S, Colditz GA, Berkman LF, Kawachi I. Caregiving and risk of coronary heart disease in U.S. women: A prospective study. Am J Prev Med. 2003; 24: 113-19.

8. Schulz R, Beach SR. Caregiving as a risk factor for mortality-the caregiver health effects study. JAMA. 1999; 282: 2215-19.

9. Montgomery RJ, Gonyea, JG, Hooyman, NR. Caregiving and the experience of subjective and objective burden. Fam Relat. 1985; 34: 19-26.

10. Vitaliano PP, Russo J, Young HM, Becker J, Maiur. The screen for caregiver burden. Gerontologist 1991; 31: 76-83.

11. Kosberg JI, Cairl RE. The cost of care index: A case management tool for screening informal care providers. Gerontologist 1986; 26: 273-78.

12. Novak M, Guest C. Application of a multidimensional caregiver burden inventory. Gerontologist 1989; 29: 798-803.

13. Beaton DE, Bombardier C, Guillemin F, Ferraz MB. Guidelines for the process of cross-cultural adaptation of self-report measures. Spine 2000; 25: 3186-91.

14. Lu L, Wang $\mathrm{L}$, Yang $\mathrm{X}$ and Feng $\mathrm{Q}$. Zarit Caregiver Burden Interview: Development, reliability and validity of the Chinese version. Psychiatry Clinic Neurosci. 2009; 63: 730-34.

15. Hirono N, Kobayashi H, Mori E. Caregiver burden in dementia: Evaluation with a Japanese version of the Zarit Caregiver Burden Interview. No To Shinkei. 1998; 50: 561-67.

16. Chattat R, Cortesi V, Izzicupo F, Letizia Del Re M, Sgarbi $\mathrm{C}$ et al. The Italian version of the Zarit Burden Interview: A validation study. Int Psychogeriatrics. 2011; 23: 797-805.

17. Yoon E, Robinson M. Psychometric properties of the Korean version of the Zarit Burden Interview (K-ZBI): Preliminary analyses. J Soc Work Res Eval. 2005; 6: 75-85.

18. Braun M, Scholz U, Hornung R, Martin M. The burden of spousal caregiving: A preliminary psychometric evaluation of the German version of the Zarit Burden Interview. Aging Ment Health. 2010; 14: 159-67.

19. Taub A, Andreoli SB, Bertolucci PH. Dementia caregiver burden: Reliability of the Brazilian version of the Zarit Caregiver Burden Interview. Cad Saude Publica. 2004; 20: 372-76.

20. Ozer N, Yurttaş A, Akyil RÇ. Psychometric evaluation of the Turkish version of the Zarit 
Burden Interview in family caregivers of inpatients in medical and surgical clinics. J Transcult Nurs. 2012; 23: 65-71.

21. Chan TSF, Lam LCW, Chiu HFK. Validation of the Chinese version of the Zarit Burden Interview. Hong Kong J Psychiatry. 2005; 15: 9-13.

22. Hébert R, Bravo G, Préville M. Reliability, validity and reference values of the Zarit Burden Interview for assessing informal caregivers of community-dwelling older persons with dementia. Canadian J Aging. 2000; 19: 494-507.

23. Ankri J, Andrieu S, Beaufils B, Grand A, Henrard JC. Beyond the global score of the Zarit Burden Interview: Useful dimensions for clinicians. Int J Geriatr Psychiatry. 2005; 20: 254-60.

24. Seng BK, Luo N, Ng WY, Lim J, Chionh HL, Goh $\mathrm{J}$, et al. Validity and reliability of the Zarit Burden Interview in assessing caregiving burden. Ann Acad Med Singapore. 2010; 39: 758-63.
25. Joel A, Sandrine A, Beatrice B, Alain G, Jean CH. Beyond the global score of the Zarit Burden Interview: Useful dimensions for clinicians. Int J Geriatr Psychiatry. 2005; 20: 254-60.

26. Knight BG, Fox LF, Chou CP. Factor structure of the Burden Interview. J Clinic Geropsychol. 2000; 6: 249-58.

27. İnci $\mathrm{FH}$, Erdem M. Bakım verme yükü ölçeğinin Türkçe'ye uyarlanması geçerlilik ve güvenilirliği [Validity and reliability of the Burden Interview and its adaptation to Turkish]. Atatürk Üniversitesi Hemşirelik Yüksekokulu Dergisi J Atatürk Univ Nursing School. 2008; 11: 85-95.

28. Paiva CE, Barroso EM, Carneseca EC, Souza CP, Santos FT, López RVM, et al. A critical analysis of test-retest reliability in instrument validation studies of cancer patients under palliative care: A systematic review. BMC Med Res Methodol. 2014; 14: 8 